FORMATION Formation emploi

Revue française de sciences sociales

112 | octobre-décembre 2010

Pêle-mêle

\title{
Évaluer une politique d'égalité professionnelle en entreprise : l'apport d'une analyse en termes de parcours
}

Assessing equal opportunities policy in companies: an analysis based on career paths

Beurteilung einer Politik der beruflichen Gleichstellung im Unternehmen:

Beitrag einer Laufbahnanalyse

Evaluar una política de igualdad profesional en la empresa : el aporte de un análisis en términos de itinerarios

Lidwine Charbeau et Guillemette de Larquier

\section{OpenEdition}

Journals

Édition électronique

URL : http://journals.openedition.org/formationemploi/3138

DOI : 10.4000/formationemploi.3138

ISSN : 2107-0946

Éditeur

La Documentation française

Édition imprimée

Date de publication : 30 décembre 2010

Pagination : 5-20

ISSN : 0759-6340

Référence électronique

Lidwine Charbeau et Guillemette de Larquier, «Évaluer une politique d'égalité professionnelle en entreprise : l'apport d'une analyse en termes de parcours », Formation emploi [En ligne], 112 | octobredécembre 2010, mis en ligne le 07 janvier 2013, consulté le 30 octobre 2020. URL : http://

journals.openedition.org/formationemploi/3138; DOI : https://doi.org/10.4000/formationemploi.3138

(c) Tous droits réservés 


\title{
Entreprise
}

\section{Évaluer une politique d'égalité professionnelle en entreprise : l'apport d'une analyse en termes de parcours}

par Lidwine Charbeau et Guillemette de Larquier ${ }^{*}$

\begin{abstract}
Une véritable politique d'égalité professionnelle devrait s'évaluer en termes de carrière et non pas seulement à coefficient égal, condition nécessaire mais non suffisante.
\end{abstract}

La réussite d'une politique de ressources humaines, quelle qu'elle soit, implique nécessairement une phase d'évaluation. La mise en place d'une politique d'égalité professionnelle n'échappe pas à cette règle : pour juger de l'évolution des inégalités entre les sexes dans l'entreprise, il convient de rendre compte des situations respectives des hommes et des femmes selon certains critères. ${ }^{1}$

De tels critères ont été établis par la loi Roudy de 1983. Elle stipule que les entreprises de plus de cinquante salariés doivent soumettre, pour avis, au comité

\footnotetext{
${ }^{1}$ Cet article est issu des travaux d'une thèse en sciences économiques et gestion, soutenue en décembre 2009 par Lidwine Charbeau, sur le thème : "Inégalités de carrières entre hommes et femmes. Une approche par les conventions d'évaluation. Analyse des trajectoires des cadres et ETAM chez un constructeur automobile ». Cette thèse a bénéficié d'un financement CIFRE (convention industrielle de formation par la recherche). Les données mobilisées dans cet article proviennent du fichier RH (ressources humaines) de l'entreprise industrielle partenaire de ce projet de thèse. Les calculs sont disponibles auprès des auteurs, tant que l'anonymat des données est respecté.
}

* Lidwine Charbeau est consultante en management et développement RH (ressources humaines). Lors de la rédaction de cet article, elle était doctorante à EconomiX, université Paris Ouest Nanterre La Défense. Elle enseigne la stratégie d'entreprise et les relations du travail. Elle travaille plus particulièrement sur les pratiques de gestion de carrières des entreprises, les critères d'évaluation des compétences. Thèmes de recherche: Évaluation des politiques $\mathrm{RH}$ en matière d'égalité professionnelle et diversité; Influence des critères d'évaluation des compétences dans l'analyse des trajectoires différenciées des hommes et des femmes en entreprise; Analyse quantitative et qualitative du plafond de verre. Elle a notamment publié : «Inégalités de salaires, inégalités d'évaluations : le cas des femmes cadres dans une grande entreprise industrielle », avec Guillemette de Larquier, in Approches institutionnalistes des inégalités en économie sociale, l'Harmattan, 2007, pp. $319-333$ 
Guillemette de Larquier est maître de conférences en économie, en détachement au Centre d'études de l'emploi depuis 2009, chercheuse associée à EconomiX, université Paris Ouest Nanterre La Défense. Elle enseigne l'économie du travail, les relations industrielles et l'économie sociale. Elle travaille plus spécifiquement sur le recrutement des entreprises et la pluralité de conventions d'évaluation du travail. Elle a codirigé deux ouvrages: Des marchés du travail équitables, Une approche comparative France Royaume-Uni, ouvrage collectif coordonné avec Ch. Bessy, F. EymardDuvernay et E. Marchal, Bruxelles, Peter Lang - Presses Interuniversitaires européennes, 2001. Approches institutionnalistes des inégalités en économie sociale (Tome 1: Évaluations; Tome 2: Politiques), Actes publics des XXV\|l| Journées de l'Association d'économie sociale, avec Ph. Batifoulier, A. Ghirardello et D. Remillon, Paris, L'Harmattan, 2007. Article récent dans le même domaine: "Assiste-ton à une transformation uniforme des carrières professionnelles vers plus de mobilité ? Une exploitation de l'enquête Histoire de vie", avec Delphine Remillon, Travail et Emploi, $n^{\circ} 113$, pp. 13-30, 2008.

d'entreprise, un rapport « sur la situation comparée des conditions générales d'emploi et de formation des femmes et des hommes » (RSC). Ce rapport fournit des chiffres sur la situation respective des femmes et des hommes en matière d'embauche, de formation, de promotion, de qualification, de classification, etc. Depuis la loi Génisson de 2001, cet outil doit également permettre d'évaluer l'impact des mesures prises par l'entreprise au cours de l'année écoulée, dans une perspective proche de celle du bilan social ${ }^{2}$, afin de servir de point d'appui à la négociation. Or, ce bilan, parce qu'il s'effectue de manière instantanée, l'année $t$ par rapport à l'année $t-1$, ne peut appréhender les différences de parcours et d'opportunités. Il risque de masquer les inégalités réelles (Cousin, 2 007).

C'est ce que nous souhaitons illustrer ici à partir de l'étude de cas réalisée par Charbeau (2009), au sein

\footnotetext{
${ }^{2}$ Comme le bilan social, le RSC est un document de référence qui rassemble un certain nombre d'indicateurs fixés par la loi (dont certains peuvent d'ailleurs être communs avec le bilan social). Il permet d'apprécier la situation comparée des hommes et des femmes dans l'entreprise en matière d'emploi, de formation, de rémunération et d'amélioration des conditions de travail et d'enregistrer les réalisations effectuées au cours de l'année écoulée et des deux années précédentes (plus si l'entreprise le juge nécessaire).
}

du département ressources humaines $(\mathrm{RH})$ d'un grand groupe français du secteur automobile (désormais nommé GFAuto). Notre objectif est d'opposer au rapport de situation comparée une analyse longitudinale plus à même de dévoiler la persistance des phénomènes inégalitaires. À partir de 2003, cette entreprise, faiblement féminisée, comme le reste du secteur, s'est engagée en faveur de l'égalité professionnelle de manière volontariste, en cohérence avec sa nouvelle stratégie industrielle. Cette entreprise, somme toute assez « exemplaire », va nous permettre de montrer qu'un diagnostic complet de la politique menée ne peut se contenter d'une évaluation des situations comparées entre hommes et femmes à coefficient égal ; ce sont les chances d'accès aux différents coefficients qu'il faut examiner. Nous nous concentrons sur la population des Employés, Techniciens et Agents de Maîtrise (ETAM). Généralement négligée par les études sur les inégalités professionnelles, cette catégorie présente pourtant l'avantage de se situer au carrefour de deux types de mobilité caractéristiques du fonctionnement d'un marché interne : les ouvriers qui deviennent ETAM et les ETAM qui deviennent cadres. Apprécier le parcours des ETAM, c'est donc pénétrer au cœur du système de promotion interne dont on sait qu'il pénalise les femmes (Dupray et Moullet, 2004 ; Gadea et Rezrari, 2004).

Dans la première partie de cette contribution, nous présentons la politique d'égalité professionnelle de GFAuto. Le bilan, qui peut être dressé à la manière du RSC officiel, est assez positif (partie 2). Néanmoins, il ne peut rendre compte de l'évolution réelle des carrières qui nécessite l'analyse d'une cohorte de salariés (partie 3). De fait, l'analyse longitudinale révèle la persistance d'inégalités de carrières entre hommes et femmes; ce que nous tentons d'expliquer dans la dernière partie à l'aide de modèles de durée.

\section{L'ÉGALITÉ PROFESSIONNELLE, PILIER DE LA NOUVELLE POLITIQUE DE L'ENTREPRISE}

Aujourd'hui, GFAuto considère l'égalité professionnelle entre hommes et femmes comme un objectif légitime, qui participe du rôle institutionnel de 
l'entreprise et de sa responsabilité sociale ; cependant, il n'en a pas toujours été ainsi. Le contexte morose qu'a connu l'entreprise entre 1978 (date de la fusion entre les deux entités qui la composent) et 1997 n'a pas favorisé l'émergence d'une politique sociale innovante. Dans le même temps, le contexte juridique de l'égalité professionnelle, peu contraignant, n'a pas engagé l'entreprise dans une vraie politique de l'emploi féminin. La nouvelle stratégie industrielle, adoptée à la fin des années 90 , va réellement changer la donne en créant des besoins importants en personnel qualifié. L'entreprise se fixe comme objectif de diversifier sa gamme de véhicules et d'augmenter massivement les volumes de production sur le marché européen et mondial, dans un contexte plus favorable. Attirer la main-d'œuvre qualifiée souhaitée nécessite alors de renouveler une stratégie RH et un système de relations sociales devenus vieillissants, mais aussi et surtout de s'ouvrir à des publics jusqu'alors négligés, parmi lesquels les femmes.

En s'adressant à des candidats de profils sociodémographiques et/ou de formation initiale différents (ouverture à des profils universitaires), GFAuto diversifie donc son recrutement. L'objectif est aussi de mettre en place une nouvelle politique de rémunération et de gestion individuelle des carrières fondée sur la performance et l'équité. Dans ce contexte, l'entreprise se saisit du thème de l'égalité professionnelle et signe, en novembre 2003, l'un des tous premiers accords d'entreprise. ${ }^{3}$

L'axe majeur de cet accord concerne la féminisation du recrutement. En 1999, le taux de féminisation est de 13,6\% (soit 11544 femmes sur un total de 85309 salariés, tous types de contrat confondus). Les responsables RH de GFAuto sont conscients de la faible féminisation des effectifs ${ }^{4}$, mais aussi de celle des filières professionnelles les plus évolutives de la conception et de la fabrication. Ils attribuent cette situation à la non-mixité des formations initiales d'une part et aux conditions de travail propres à certains secteurs d'autre part. Or, l'entreprise « reconnaît dans la mixité professionnelle une source de complémentarité, d'équilibre

\footnotetext{
${ }^{3}$ Le recrutement d'un étudiant en thèse CIFRE à l'origine de cette étude a d'ailleurs été formalisé dans l'accord d'entreprise (article 3.9).

${ }^{4}$ Féminisation qui est cependant comparable à celle des entreprises du secteur.
}

social et d'efficacité économique » (préambule de l'accord). La mixité est donc valorisée en soi. L'essentiel de la politique mise en place vise alors à renforcer la mixité des métiers. Pour ce faire, on encourage, en amont, l'arrivée des jeunes femmes : en identifiant les postes qualifiés et les métiers encore mal adaptés au personnel féminin; en comparant la part des femmes recrutées à la part des femmes diplômées de chaque filière de formation initiale, ainsi que la part des femmes recrutées à la part des femmes reçues en entretien; enfin en intervenant en amont des processus de recrutement, en coopération avec l'Éducation nationale, pour favoriser l'orientation des jeunes filles dans des filières industrielles et les accueillir en stage.

Une politique active de recrutement est inutile si les femmes récemment intégrées dans l'entreprise ne peuvent prétendre aux mêmes opportunités de carrière que leurs homologues masculins. L'accord prévoit ainsi qu'au-delà de l'égalité salariale à coefficients identiques, la répartition des augmentations individuelles et des promotions devra refléter la répartition hommes-femmes de la population d'origine. Les principes de non-discrimination et d'égalité de traitement en matière de promotion sont également rappelés : « les mêmes critères de détection des potentiels internes seront utilisés pour les femmes et pour les hommes. Ils sont fondés exclusivement sur le seul exercice des compétences et donc de l'efficacité et de la performance. »Il est prévu que, pour parvenir à une meilleure représentation des femmes dans les postes traditionnellement masculinisés, des plans d'action en termes de formation, de mobilité et d'orientation professionnelle seront mis en place. GFAuto s'engage également à ce que le congé maternité soit sans incidence sur le déroulement de carrière.

Enfin, partant du principe que l'amélioration des conditions de travail facilite la féminisation de l'emploi, l'accord stipule qu'un état des lieux des conditions de travail doit être réalisé dans tous les établissements, en lien avec les Comités d'Hygiène, de Sécurité et des Conditions de Travail. L'aménagement des postes de travail, l'adaptation des locaux sociaux et des infrastructures dédiées au personnel féminin et le développement de solutions (aménagements des horaires individuels ou autres dispositifs) sont présentés comme autant de dispositifs volontaristes en faveur des femmes visant à concilier vie personnelle et vie professionnelle. 


\section{L'ÉGALITÉ PROFESSIONNELLE EN BONNE VOIE ?}

Pour mesurer les effets de sa politique, GFAuto dispose d'outils d'évaluation qui, loin d'être arbitraires, ont été fixés par la loi. Ils sont rassemblés dans le rapport annuel de situation comparée sur les conditions générales d'emploi des femmes et des hommes. Ils peuvent bien sûr être complétés par toutes les informations jugées pertinentes par l'entreprise. Aussi, si le RSC est un outil encore trop peu utilisé par les entreprises (Grésy, 2009), il reste un document de référence sur la façon dont sont appréhendées les inégalités hommes-femmes dans le cadre de l'entreprise. Il sert en outre de point d'appui aux négociations collectives et aux mesures correctives. Nous proposons donc, à la manière du RSC, de présenter la situation comparée des femmes et des hommes ETAM à la date du 31/12/2006. Il s'agit d'établir un premier bilan trois ans après la signature de l'accord sur le développement de l'emploi féminin.

\section{Les effectifs ETAM :} des efforts de mixité

Signe de la croissance qu'a connue GFAuto à la fin des années 90 et au début des années 2000, la population des ETAM s'est fortement accrue. En dix ans, cette population est passée de 10948 à 17150 individus. Elle représente près d'un cinquième des effectifs de l'entreprise en 2006. Toutefois, la part des femmes a peu évolué en proportion : tandis qu'elles représentaient $18,9 \%$ des effectifs ETAM en 1996, leur part est de $20,3 \%$ en 2006 .

Outre la féminisation générale, nous l'avons dit, c'est la mixité professionnelle que l'accord d'entreprise valorise en soi. On part d'une situation où les ETAM, hommes et femmes, se répartissent très inégalement entre les quatre grands groupes de métiers (deux étant très fortement masculinisés : agents de maîtrise et techniciens). C'est là le signe d'une segmentation horizontale et d'une concentration traditionnelle des emplois féminins bien décrite par Obka (2004). Or, depuis la fin des années 90 , le levier du recrutement

\section{Graphique 1}

Évolution du taux de féminisation des ETAM (employés, techniciens, agents de maîtrise) par famille professionnelle entre 1996 et 2006

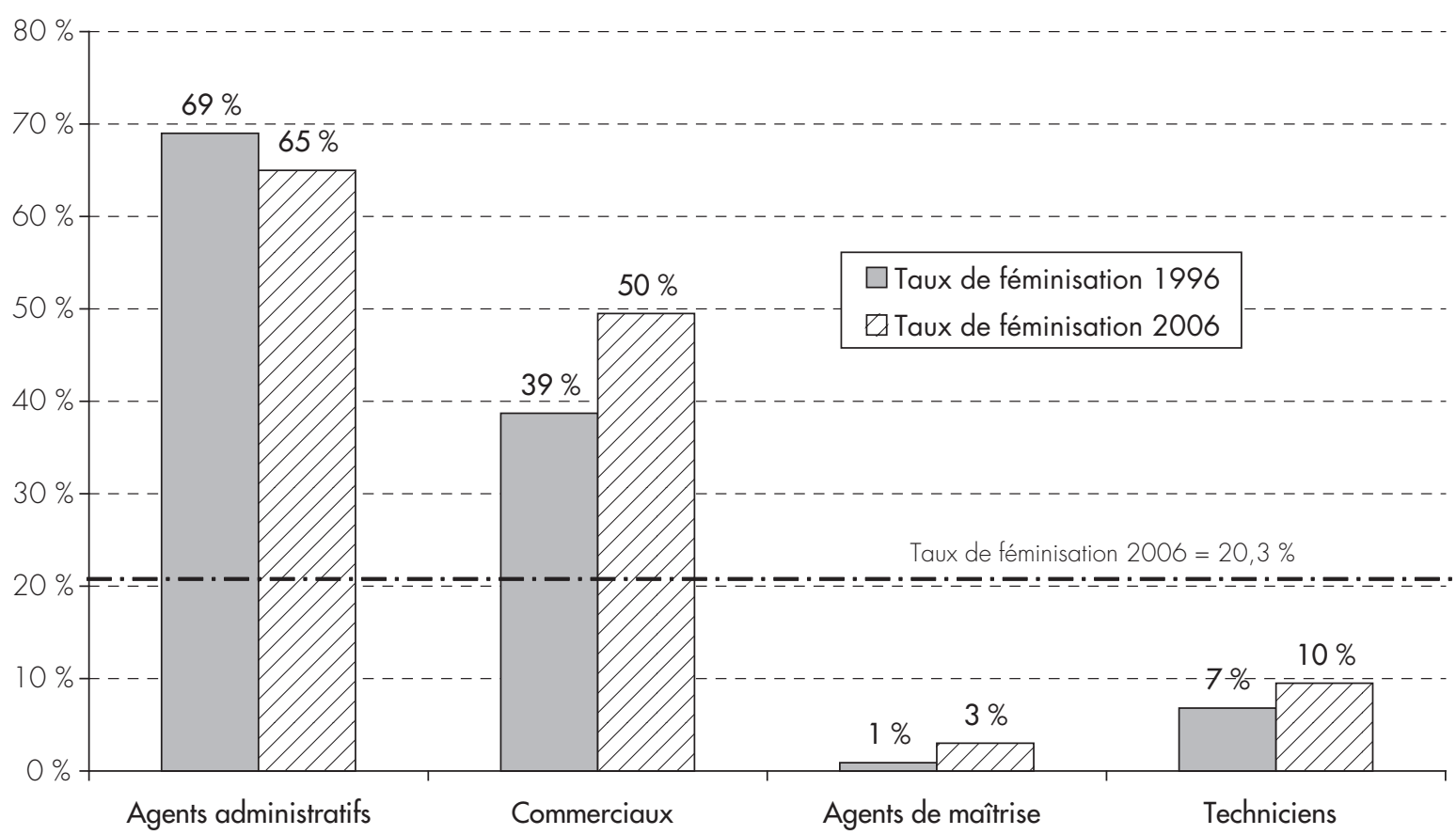

Source : fichiers RH de GFAuto. 
a peu joué pour les femmes ETAM, contrairement à leurs homologues cadres étudiées par ailleurs (Charbeau et Larquier, 2007). Néanmoins, l'évolution de la répartition des sexes au sein des familles professionnelles au cours de la période suggère que des efforts de mixité des emplois ont bien été engagés ${ }^{5}$ (Graphique 1), même si les femmes restent fortement concentrées dans les fonctions d'appui : agents administratifs et commerciaux. Le RSC de 2006 se satisfait ainsi d' "une évolution positive du pourcentage de femmes présentes dans les métiers qui étaient essentiellement masculins, [...] ces métiers offr[ant] souvent des perspectives de carrière ».

\section{Une politique salariale égalitaire}

Sur le plan de la politique salariale, le bilan est particulièrement positif. Comme l'illustre le tableau 1, établi à partir du RSC de 2006, "à coefficient identique, le salaire moyen est équivalent entre les hommes et les femmes ». Ici, nous avons fait apparaître les rapports de salaires moyens entre hommes et femmes selon le coefficient (dans le RSC sont mentionnés les niveaux absolus). Conformément à la convention collective de la métallurgie, dont dépend GFAuto, 14 coefficients, regroupés en cinq niveaux, classent les ETAM de façon croissante : niveau II (coefficients 170, 180, 190), niveau III (coefficients 215, 225, 240), niveau IV $(255,270,285)$, niveau V $(305,320,335,365 /$ K92). Du coefficient, multiplicateur de l'appointement minimum défini entre les partenaires sociaux, on déduit l'échelon correspondant et le salaire de base. Ce dernier est modulé par l'ancienneté et une éventuelle augmentation individuelle (AI), essentiellement fonction de la bonne tenue du poste évaluée tous les ans par le supérieur hiérarchique ${ }^{6}$. Cette règle semble s'appliquer sans introduire de biais de genre. On observe néanmoins que la situation est d'autant plus favorable aux femmes qu'elles sont moins nombreuses dans leur coefficient, ce qui est plus souvent le cas au sein des coefficients élevés. Il y aurait pour les femmes une prime à la non-mixité (Fortino, 2002).

\footnotetext{
5 Y compris en affectant plus d'hommes parmi les agents administratifs.

${ }^{6}$ Dans le tableau 1, les primes ne sont pas prises en compte. Par ailleurs, nous avons exclu les coefficients pour lesquels les effectifs étaient inférieurs à 10 .
}

Tableau 1

Rapports de salaires de base (hors primes) moyens entre les hommes et les femmes selon le coefficient en 2006

\begin{tabular}{|c|c|c|}
\hline Coefficients & $\begin{array}{c}\text { Rapport de salaires } \\
\text { moyens }\end{array}$ & $\begin{array}{c}\text { Taux de } \\
\text { féminisation }\end{array}$ \\
\hline 215 & 1,00 & $26,3 \%$ \\
225 & 1,01 & $37,2 \%$ \\
240 & 1,01 & $41,6 \%$ \\
255 & 0,99 & $32,6 \%$ \\
270 & 0,99 & $24,9 \%$ \\
285 & 0,98 & $21,4 \%$ \\
305 & 0,99 & $16,3 \%$ \\
335 & 0,98 & $14,3 \%$ \\
\hline Ensemble & 1,02 & 20,3 \\
\hline
\end{tabular}

Lecture : En 2006, le salaire moyen d'un homme ETAM (employés, techniciens, agents de maîtrise) dans le coefficient 240 est égal à 1,01 fois celui d'une femme dans le même coefficient. Champ : l'ensemble des ETAM en 2006.

Source : RSC et fichiers RH de GFAuto.

La répartition des effectifs féminins au sein des coefficients de la catégorie ETAM apparaît donc être l'enjeu majeur : l'essentiel des différences entre les hommes et les femmes semble se jouer dans l'accès aux coefficients les plus élevés. Or, de nouveau, les indicateurs du RSC de 2006 permettent à GFAuto de formuler un diagnostic flatteur: "pour les ETAM, la proportion de femmes ayant bénéficié d'un changement de coefficient (15,3\%) est supérieure à celle des hommes $(14,3 \%)$ ». À l'aune du document de référence qu'est le RSC, l'égalité professionnelle semble donc en bonne voie chez GFAuto.

\section{LE RAPPORT DE SITUATION COMPARÉE PEINE À ÉVALUER LES INÉGALITÉS DE PARCOURS}

Nous souhaitons montrer ici l'incomplétude du RSC pour évaluer une politique d'égalité professionnelle qui n'aurait aucun sens en l'absence d'opportunités de carrière équivalentes. Le RSC fournit une photographie de l'entreprise à une date $t$, résultat bien sûr des carrières qui s'y déroulent mais aussi des mouvements de sortie et d'entrée de main-d'œuvre. Or, la politique 
de recrutement de GFAuto a été très active les années précédant 2006 ; les indicateurs du RSC en rendent d'ailleurs compte, certes de manière légitime, mais sans distinguer ce qui relève de la politique volontariste de recrutement de femmes de ce qui relèverait plutôt d'une amélioration de leurs carrières.

Pour évaluer les effets de la politique de l'entreprise sur les carrières, il faut en toute rigueur suivre une cohorte de salariés, femmes et hommes, et leurs parcours. Ce que l'on donnera alors à voir de GFAuto ne sera pas plus « juste » ou « correct » mais complémentaire; il y aura en effet un biais de sélection assumé consistant à considérer uniquement les ETAM qui sont présents depuis plusieurs années et qui ne sont pas sortis. Dans le cas de GFAuto, grand marché interne où les sorties autres que les retraites sont marginales, cette analyse sur données longitudinales, en complément de l'approche en coupe instantanée du RSC, nous semble particulièrement pertinente. En effet, GFAuto étant un groupe où les carrières longues sont la norme, mesurer les effets de la politique d'égalité professionnelle sur une cohorte de salariés semble techniquement possible (constitution d'un échantillon suffisamment large). En outre, cela vise le cœur même du problème, la construction progressive des inégalités entre hommes et femmes dans l'entreprise.

\section{Construire la cohorte : le parcours professionnel sur dix ans}

Nous retenons l'ensemble des salariés ETAM en 1996 encore présents dans l'entreprise en 2006, qu'ils soient restés ETAM ou devenus cadres. La convention collective de la métallurgie prévoit automatiquement un passage de deux coefficients en 18 mois pour les ETAM entrés avec un niveau de diplôme $\mathrm{Bac}+2$. Pour neutraliser cet effet, nous avons travaillé sur la population ETAM de 1996 mais présente depuis 1994. Nous bénéficions alors du parcours professionnel de 10585 personnes, dont $19,4 \%$ de femmes, sur une période de dix ans. Parmi cette population d'ETAM, 7478 individus, soit environ $70 \%$ de notre population, sont restés ETAM, les autres sont devenus cadres sur la période.

Dans un premier temps, nous présentons l'information que l'on peut tirer de notre cohorte, sous forme de tableaux synthétiques, de même nature que ceux que l'on trouve dans un RSC. Il s'agit de proposer des indicateurs simples dont une entreprise pourrait se saisir ; la difficulté principale pour l'entreprise étant plutôt la constitution d'un fichier RH ayant la mémoire du passé pour construire la cohorte ${ }^{7}$. Dans la dernière partie, nous mobiliserons des modèles de durée. Nous viserons alors à expliquer les inégalités de carrières que l'on aura mises en évidence. Dans les deux cas, nous ne retenons que les ETAM dont le coefficient d'origine est supérieur ou égal à 240 (pour une question d'effectif). Mais chaque exercice nécessitera de « retailler » la cohorte.

\section{Des retards de carrière occultés par le rapport de situation comparée}

Nous allons ici comparer la situation des femmes et des hommes de la même cohorte à dix ans d'intervalle, en 1996 et 2006. Ce sont donc nos données qui sont longitudinales et non notre analyse, qui rapproche deux photographies instantanées. Pour comparer sur une base relativement simple des femmes et des hommes « comparables », nous retenons deux critères : le coefficient et l'âge. Nous découpons l'échantillon initial en cinq sous-cohortes, par tranche d'âge de cinq ans, retenant au total les ETAM ayant entre 25 et 49 ans en 1996. Nous pouvons alors raisonner en termes de générations.

Le tableau 2 est construit en reproduisant la logique du RSC, c'est-à-dire en raisonnant à coefficient donné. Le constat d'une égalité de traitement est préservé ; mieux encore, à coefficient donné, les rapports salariaux entre les hommes et les femmes ETAM, qui étaient déjà faiblement inégalitaires en 1996, se sont encore réduits en 2006, quand ils ne sont pas favorables aux femmes.

\footnotetext{
${ }^{7}$ Ce qui était le cas de GFAuto qui ne disposait pas de fichier du personnel disponible et "prêt à l'emploi ». Cela tient à l'histoire du groupe, dont la politique $\mathrm{RH}$ commune est récente, mais aussi à la façon dont les données sont classées au sein des systèmes d'information RH. Les informations relatives aux carrières (salaire, position dans la classification, promotion), mais aussi celles liées aux données personnelles (formation initiale, situation familiale, etc.) relèvent de différents « univers d'informations ». Les données disponibles ont donc été extraites des systèmes d'information RH sous la forme de requêtes multiples réalisées sous Business Object. Elles ont ensuite été croisées à partir des matricules individuels, puis ont été rassemblées sous la forme d'une base de données anonyme.
} 
Tableau 2

Rapports de salaires de base (hors primes) moyens

entre les hommes et les femmes selon le coefficient en 1996 et 2006

\begin{tabular}{|c|c|c|c|c|c|c|c|c|}
\hline Année Coef. & 240 & 255 & 270 & 285 & 305 & 335 & 365 & Cadres \\
\hline 1996 & 1,02 & 1,01 & 1,01 & 0,99 & 0,99 & 1,00 & 1,01 & - \\
\hline 2006 & 1,02 & 1,01 & 1,00 & 0,99 & 0,98 & 0,98 & - & 1,01 \\
\hline Taux de variation & $-0,59 \%$ & $-0,01 \%$ & $-1,18 \%$ & $0,82 \%$ & $-0,92 \%$ & $-1,81 \%$ & & \\
\hline
\end{tabular}

Lecture : En 1996, le salaire moyen d'un homme ETAM (employés, techniciens, agents de maitrise) dans le coefficient 270 est égal à 1,01 fois celui d'une femme dans le même coefficient. Dix ans plus tard, au sein du même coefficient, le rapport est égal à 1,00. Il a donc diminué de $1,18 \%$ au profit des femmes.

Champ : les ETAM présents en 1996 et 2006 ayant entre 25 et 49 ans en 1996.

Source : fichiers RH de GFAuto.

Tableau 3

Rapports de salaires de base (hors primes) moyens entre les hommes et les femmes en 1996 et 2006 selon la génération

\begin{tabular}{|c|c|c|c|c|c|}
\hline Année Coef. & $1967-71$ & $1962-66$ & $1957-61$ & $1952-56$ & $1947-51$ \\
\hline 1996 & 0,98 & 1,01 & 1,03 & 1,04 & 1,06 \\
\hline 2006 & 1,02 & 1,06 & 1,06 & 1,06 & 1,11 \\
\hline Taux de variation & $3,90 \%$ & $5,22 \%$ & $3,25 \%$ & $1,94 \%$ & $4,31 \%$ \\
\hline
\end{tabular}

Lecture : Les hommes nés entre 1957 et 1961 ont, en 1996, un salaire moyen égal à 1,03 fois celui de leurs contemporaines ; 10 ans plus tard, il est égal à 1,06 fois celui de ces mêmes femmes. Le rapport a donc augmenté de 3,25\% au profit des hommes de cette génération.

Champ : les ETAM (employés, techniciens, agents de maîtrise) présents en 1996 et 2006 ayant entre 25 et 49 ans en 1996.

Source : fichiers RH de GFAuto.

Le tableau 3, en revanche, où les calculs sont réalisés non plus en fonction du coefficient mais de la génération, nourrit un autre diagnostic. Entre 1996 et 2006, les rapports de salaires moyens par génération ont plutôt augmenté au profit des hommes. De plus, la situation de départ est d'autant plus défavorable aux femmes que la génération est ancienne; ce tableau met justement en évidence le résultat cumulatif du phénomène. Si à coefficient égal, les femmes sont traitées comme les hommes, il apparaît néanmoins qu'en début, en milieu ou en fin de carrière, les femmes ETAM ont toutes connu des progressions salariales plus lentes que celles de leurs contemporains masculins. Elles n'ont pas les mêmes chances d'atteindre ce «coefficient égal». Autrement dit, l'inégale répartition des hommes et des femmes au sein des coefficients, à génération donnée, s'amplifie avec le temps.

Pour conserver le raisonnement en termes de coefficient égal, qui structure la mise en forme de l'information dans le RSC, et néanmoins évaluer la dimension dynamique des inégalités, il conviendrait donc de recalculer les salaires moyens ou rapports de salaires moyens en fonction du coefficient initial et non plus d'arrivée. Le tableau 2 bis permet ainsi d'évaluer la situation comparée d'hommes et de femmes partis du même coefficient et non plus la situation comparée de personnes arrivées au même point dans leur carrière (logique du tableau 2). En changeant ainsi l'angle de vue, il ressort que les hommes partant du même coefficient que les femmes en 1996 ont eu une progression salariale légèrement plus importante qu'elles : tous les rapports ont évolué en leur faveur; alors que le tableau 2 montrait plutôt que les femmes ayant eu la même carrière que les hommes (arrivées au même coefficient) étaient aussi bien traitées, si ce n'est mieux. Ces deux résultats ne sont pas incompatibles ; cependant, alors que le tableau 2 évalue la politique de rémunération basée sur le coefficient (qui apparaît neutre au genre), le tableau 2 bis évalue la politique de promotion au sein des coefficients (en dévoilant un biais léger mais persistant lié au genre). 
Tableau 2bis

Rapports de salaires de base (hors primes) moyens

entre les hommes et les femmes selon le coefficient de départ en 1996

\begin{tabular}{|c|c|c|c|c|c|c|c|}
\hline Coef. & 240 & 255 & 270 & 285 & 305 & 335 & 365 \\
\hline Salaires 1996 & 1,02 & 1,01 & 1,01 & 0,99 & 0,99 & 1,00 & 1,01 \\
\hline Salaires 2006 & 1,03 & 1,03 & 1,02 & 1,02 & 1,02 & 1,05 & 1,02 \\
\hline
\end{tabular}

Lecture : En 2006, le salaire moyen d'un homme ETAM (employés, techniciens, agents de maitrise), qui était dans le coefficient 240 en 1996 , est égal à 1,03 fois celui d'une femme partant du même coefficient en 1996.

Champ : les ETAM présents en 1996 et 2006 ayant entre 25 et 49 ans en 1996.

Source : fichiers RH de GFAuto.

Finalement, l'égalité professionnelle ne semble pas acquise. Si la politique de rémunération de GFAuto avait été simplement sexiste, le RSC aurait suffi à le révéler. Or, en l'absence d'un sexisme primaire, il demeure des inégalités que le RSC ne peut pas dévoiler, faute d'un questionnement sur les carrières.

\section{LA PROMOTION DES ETAM À L'AUNE DU MODĖLE DE DISPONIBILITÉ}

Nous venons de mettre en évidence la moindre progression moyenne des femmes dans l'entreprise GFAuto. Par conséquent, ce ne sont pas les différences de salaires qu'il faut chercher à expliquer « toutes choses égales par ailleurs », version savante de « à coefficient égal », mais les différences de promotion ${ }^{8}$. Pour ce faire, nous mobilisons ici des modèles de durée.

\section{Deux types de promotion}

Une promotion est une transition entre deux états. Un modèle de durée explique la durée dans le premier

\footnotetext{
${ }^{8}$ Un certain nombre de travaux économiques portent ainsi avant tout sur les promotions, suggérant que les différences de promotion participent de manière essentielle à l'explication des différences de salaires. Certaines de ces études ont été réalisées à partir d'enquêtes nationales et/ou transversales à un grand nombre d'entreprises (Mc Cue, 1996 ; Pargamit et Veum, 1999; Coob-Clark, 2001 ; Booth et alii, 2003 ; Blau et DeVaro, 2007). Mais la plupart d'entre elles, comme notre étude, analysent des données d'entreprise ou de secteur professionnel pour évaluer, au sein des organisations (Gerhart et Milkovitch, 1989; Dupray et Moullet, 2004 ; Cousin, 2007) ou des professions (Ranson et Reeves, 1996 ; Pekkarinen et Vartiainen, 2004 ; Carrere et Sabatier, 2004 ; Carrere, Sabatier et Mangematin, 2006), les chances de promotion relatives des hommes et des femmes.
}

état ; dans notre cas, la durée passée dans un coefficient avant d'être promu. Carrere et Sabatier (2004) ou Carrere, Sabatier et Mangematin (2006) ont de la même façon utilisé cette méthode pour expliquer le déroulement de la carrière des femmes universitaires. Il s'agissait d'identifier les facteurs qui accéléraient ou au contraire ralentissaient l'accès au statut de professeur des universités.

Nous disposons de l'information sur les coefficients à partir de 1996. Toutefois, nous ignorons depuis quand les individus sont dans le coefficient de 1996 : on parle de censure à gauche. Pour contourner la difficulté, nous étudions les évolutions individuelles en repérant la date, après 1996, où les individus accèdent à un nouveau coefficient qui sera le coefficient initial à partir duquel on préfère raisonner. Ainsi notre analyse ne porte-t-elle que sur les individus qui ont réellement connu au moins une transition avant 2006, nécessaire à la détermination du coefficient initial. Nous testons la durée dans ce coefficient avant une éventuelle seconde transition ${ }^{9}$ jusqu'en 2006.

Les ETAM constituent une population susceptible de connaître deux types de promotion : vers un coefficient ETAM supérieur et vers un statut cadre. Nous allons étudier les deux transitions de manière distincte. Pour le passage cadre, nous considérons des individus qui ont un niveau de coefficient suffisant pour pouvoir prétendre devenir cadre en moins de dix ans. Nous avons donc retenu les ETAM qui ont un coefficient initial supérieur ou égal à 305 . On estime alors la durée avant de devenir cadre, sans tenir compte du fait que l'individu a pu passer par un autre coefficient

\footnotetext{
9 Elle peut ne pas être survenue au moment de la fin de l'observation (au 31/12/2006). Dans ce cas, les données sont dites censurées à droite ; la particularité d'un modèle de durée est de pouvoir gérer cette censure-là
} 
ETAM entre-temps. Pour la vitesse de progression au sein des coefficients ETAM, nous avons à l'inverse écarté toutes les personnes dont le coefficient initial est supérieur ou égal à 305, à partir duquel c'est plutôt la sortie de la catégorie ETAM qui est en jeu. Finalement, nous comptons 3618 individus ETAM qui resteront ETAM et 3823 ETAM susceptibles de devenir cadres. Les deux populations sont décrites dans le tableau 4, selon le sexe. La durée moyenne dans un coefficient varie entre 3,5 et 4 ans. Quant au passage cadre, il exige en moyenne 6 ans depuis le coefficient 305 . Une fois atteint le coefficient 365 , le passage au statut cadre est une formalité dans l'année qui suit ; c'est pourquoi nous allons concentrer notre attention sur ceux qui partent d'un coefficient initial 305 ou 335.

Tableau 4

Description des populations des modèles de durées (en \%)

\begin{tabular}{|c|c|c|c|c|}
\hline & \multicolumn{2}{|c|}{ Les ETAM restant ETAM } & \multicolumn{2}{|c|}{$\begin{array}{l}\text { Les ETAM susceptibles de } \\
\text { passer Cadre }\end{array}$} \\
\hline & $\mathrm{H}$ & $\mathrm{F}$ & $\mathrm{H}$ & $\mathrm{F}$ \\
\hline Promus sur la période étudiée & 84,4 & 72,0 & 32,3 & 26,1 \\
\hline $\begin{array}{l}\text { Classe d'âge en 1996: } \\
<30 \text { ans } \\
30-34 \text { ans } \\
\text { 35-39 ans } \\
\text { 40-44 ans } \\
\text { 45-49 ans } \\
\text { 50-54 ans }\end{array}$ & $\begin{array}{c}2,6 \\
14,3 \\
18,7 \\
28,0 \\
26,6 \\
9,9 \\
100 \% \\
\end{array}$ & $\begin{array}{l}2,0 \\
9,2 \\
14,0 \\
29,0 \\
28,9 \\
17,0 \\
100 \% \\
\end{array}$ & $\begin{array}{l}4,9 \\
26,4 \\
19,4 \\
14,8 \\
21,2 \\
13,4 \\
100 \% \\
\end{array}$ & $\begin{array}{l}4,9 \\
20,7 \\
16,4 \\
17,7 \\
24,1 \\
16,2 \\
100 \% \\
\end{array}$ \\
\hline $\begin{array}{l}\text { Diplôme : } \\
\text { BEPC/CEP } \\
\text { CAP/BEP } \\
\text { Bac } \\
\text { Bac }+2 \\
\text { Bac }+3 \text { et plus }\end{array}$ & $\begin{array}{c}8,5 \\
44,6 \\
39,9 \\
6,3 \\
0,7 \\
100 \% \\
\end{array}$ & $\begin{array}{l}17,7 \\
49,6 \\
27,5 \\
4,3 \\
1,0 \\
100 \% \\
\end{array}$ & $\begin{array}{c}4,5 \\
22,1 \\
16,1 \\
53,5 \\
3,8 \\
100 \%\end{array}$ & $\begin{array}{l}8,9 \\
18,3 \\
18,8 \\
51,7 \\
2,3 \\
100 \%\end{array}$ \\
\hline A été recruté comme ouvrier & 68,7 & 19,5 & 28,1 & 11,2 \\
\hline $\begin{array}{l}\text { Coefficient initial : } \\
240 \\
255 \\
270 \\
285\end{array}$ & $\begin{array}{c}3,7 \\
19,3 \\
35,8 \\
41,2\end{array}$ & $\begin{array}{l}18,4 \\
26,0 \\
31,4 \\
24,2\end{array}$ & & \\
\hline $\begin{array}{l}305 \\
335\end{array}$ & $100 \%$ & $100 \%$ & $\begin{array}{l}48,4 \\
51,6 \\
100 \% \\
\end{array}$ & $\begin{array}{l}55,1 \\
44,9 \\
100 \% \\
\end{array}$ \\
\hline $\begin{array}{l}\text { Famille professionnelle initiale: } \\
\text { Agents administratifs } \\
\text { Commerciaux } \\
\text { Agents de Maîtrise } \\
\text { Techniciens }\end{array}$ & $\begin{array}{l}9,0 \\
1,0 \\
27,6 \\
62,4 \\
100 \%\end{array}$ & $\begin{array}{l}76,8 \\
4,7 \\
1,2 \\
17,3 \\
100 \%\end{array}$ & $\begin{array}{l}5,2 \\
1,2 \\
13,9 \\
79,8 \\
100 \%\end{array}$ & $\begin{array}{l}62,4 \\
4,2 \\
1,0 \\
32,5 \\
100 \%\end{array}$ \\
\hline
\end{tabular}




\begin{tabular}{|c|c|c|c|c|}
\hline \multicolumn{5}{|c|}{ Tableau 4 (suite) } \\
\hline & \multicolumn{2}{|c|}{ Les ETAM restant ETAM } & \multicolumn{2}{|c|}{$\begin{array}{l}\text { Les ETAM susceptibles de } \\
\text { passer Cadre }\end{array}$} \\
\hline & $\mathrm{H}$ & $\mathrm{F}$ & $\mathrm{H}$ & $\mathrm{F}$ \\
\hline Établissement situé en Région parisienne & 59,6 & 42,1 & 61,7 & 38,4 \\
\hline $\begin{array}{l}\text { Nombre d'enfants: } \\
\text { Aucun } \\
\text { Un seul } \\
\text { Deux } \\
\text { Trois et plus }\end{array}$ & $\begin{array}{l}17,0 \\
20,6 \\
43,0 \\
19,6 \\
100 \%\end{array}$ & $\begin{array}{c}14,1 \\
33,2 \\
45,7 \\
7,1 \\
100 \%\end{array}$ & $\begin{array}{l}25,2 \\
21,9 \\
38,3 \\
14,6 \\
100 \%\end{array}$ & $\begin{array}{c}22,6 \\
30,8 \\
40,9 \\
5,7 \\
100 \%\end{array}$ \\
\hline $\begin{array}{l}\text { Sur la période précédent la promotion: } \\
\text { Prise d'un temps partiel long (> } 6 \text { mois) } \\
\text { Naissance d'un enfant } \\
\text { Mobilité régionale } \\
\text { Changement de famille professionnelle }\end{array}$ & $\begin{array}{l}0,0 \\
9,3 \\
1,2 \\
6,0 \\
\end{array}$ & $\begin{array}{c}11,8 \\
6,9 \\
0,8 \\
2,7 \\
\end{array}$ & $\begin{array}{c}0,0 \\
18,2 \\
2,6 \\
11,4 \\
\end{array}$ & $\begin{array}{c}22,4 \\
13,3 \\
1,7 \\
10,8 \\
\end{array}$ \\
\hline$N$ & 2701 & 917 & 3297 & 526 \\
\hline
\end{tabular}

Champ : Pour la première population, ne sont considérés que les ETAM ayant un coefficient inférieur à 305 ; pour la seconde, ceux ayant au contraire un coefficient supérieur à 305 et donc susceptibles d'être promus cadres, ce qui explique les cellules grisées du tableau.

Lecture : La population des ETAM (employés, techniciens, agents de maitrise) retenue pour étudier les promotions au sein des coefficients ETAM entre 1997 et 2006, est composée de 2701 hommes et 917 femmes. 84,4 \% des hommes ont connu au moins une promotion pendant la période, ainsi que $72,0 \%$ des femmes. La seconde population extraite pour étudier le passage au statut cadre est composée de 3297 hommes et 526 femmes ; 32,3\% d'entre eux sont devenus cadres entre 1997 et 2006 ainsi que $26,1 \%$ d'entre elles. On note que la seconde population est plus jeune et plus diplômée.

Sigles : BEPC : brevet des collèges ; CEP : certificat d'études primaires. CAP : certificat d'aptitude professionnelle ; BEP : brevet d'études professionnelles.

\section{Les déterminants individuels de la vitesse de promotion}

Nous cherchons à mesurer l'effet d'un certain nombre de variables individuelles (sexe, âge, diplôme, situation familiale, nombre d'enfants) et professionnelles (recrutement en tant qu'ouvrier ou ETAM, coefficient initial, famille professionnelle, localisation de l'établissement $)^{10}$ sur la vitesse avec laquelle survient chaque type de promotion. En particulier, nous avons isolé l'ensemble des événements (naissance d'un enfant, passage à un temps partiel long, changement de famille professionnelle, mutations géographiques) qui peuvent intervenir avant la transition et avoir un effet sur la carrière. Cela nous amène à estimer des modèles de durée semi-paramétriques de type

${ }^{10}$ Dans cette liste n'apparaît pas l'ancienneté. Compte tenu de la forte corrélation de l'âge et de l'ancienneté dans ce marché interne, il fallait faire un choix entre les deux variables pour le modèle de durée. Après essais, l'âge est apparu comme la variable la plus pertinente. modèle de $\mathrm{Cox}^{11}$ qui permettent justement de prendre en compte des caractéristiques individuelles susceptibles de se modifier au cours du temps (Lelièvre et Bringé, 1998).

${ }^{11}$ On cherche à estimer l'influence de caractéristiques (le sexe, l'âge, etc., notées $\mathrm{X}$ ) sur la probabilité qu'un événement précis (ici une promotion) se produise pendant un intervalle de temps $[t, t+\Delta]$ sachant que l'événement ne s'est pas réalisé auparavant. Il s'agit du risque instantané $\lambda(t, X)$. Dans un modèle de Cox, le risque instantané $\lambda(t, X)$ est le produit d'un risque de base $\lambda_{0}(t)$ et d'une fonction indépendante du temps $\varphi(X ; \beta)$, où $\beta$ sont les paramètres à estimer. On dit que le modèle est semi-paramétrique car on ne spécifie pas la fonction du risque de base $\lambda_{0}(t)$ (ce qui permet de s'affranchir d'hypothèses sur la relation entre le temps et le risque de base), alors que la fonction $\varphi$ est spécifiée comme étant exponentielle. Dans ces conditions, le résultat de l'estimation (l'exponentiel du paramètre $\beta_{\mathrm{i}}$ de la caractéristique $\mathrm{X}_{\mathrm{i}}$ ) peut s'interpréter comme un rapport de risques instantanés, à savoir un rapport avec au dénominateur le risque instantané de la population de référence et, au numérateur, le risque instantané de la population qui ne diffère de la première que par la caractéristique $\mathrm{X}_{\mathrm{i}}$ dont on teste l'effet « toutes choses égales par ailleurs ». Étant donné les hypothèses de spécification, ces rapports ne dépendent pas du temps. C'est pourquoi le modèle de Cox est également qualifié de modèle à risques proportionnels : le risque n'est pas forcément constant, mais il évolue pour tous de la même manière. En revanche, on peut introduire dans le modèle des caractéristiques $\mathrm{X}_{\mathrm{i}}$ dépendantes du temps, comme la naissance d'un enfant pendant la période étudiée (Lelièvre et Bringé, 1998). 
Nous avons dû créer une variable croisant le sexe et la prise d'un temps partiel long sur la période. En effet, on observe qu'il s'agit d'une pratique exclusivement féminine : un cinquième de notre population féminine ETAM de 1996 a eu recours à un temps partiel long (supérieur à six mois) sur la période contre moins d'1 \% des hommes. Face à cette corrélation excessive, notre solution a consisté à écarter les quelques hommes qui ont pu prendre un temps partiel et à distinguer les femmes selon qu'elles ont connu ou non une période supérieure à six mois en temps partiel. Enfin, il a semblé intéressant d'estimer les durées non seulement sur l'ensemble des ETAM, mais également au sein des deux familles professionnelles les plus importantes, à savoir les Agents administratifs et les Techniciens. La question posée est alors celle d'un effet positif ou négatif de la mixité : l'effet sexe joue-t-il également, et si non dans quel sens, selon que la population est très féminisée (cas des agents administratifs : environ $70 \%$ ) ou très peu (cas des techniciens : moins de $10 \%$ ) ?

Les résultats figurent dans le tableau 5, où apparaissent les rapports de risques instantanés significatifs à un seuil inférieur à $5 \%$. Un rapport supérieur à 1 signifie que, toutes choses égales par ailleurs, le risque de promotion est augmenté (donc la durée est plus courte), un rapport inférieur à 1 signifie que ce risque est diminué (donc la durée est plus longue).

Tableau 5

Estimations des modèles de durées

\begin{tabular}{|c|c|c|c|c|c|c|}
\hline & \multicolumn{3}{|c|}{$\begin{array}{c}\text { Changer de coefficient au sein } \\
\text { des ETAM } \\
\text { (entre } 1997 \text { et } 2006 \text { ) (1) }\end{array}$} & \multicolumn{3}{|c|}{$\begin{array}{c}\text { Devenir cadre à partir des coefficients } \\
305 \text { et } 335 \\
\text { (entre } 1997 \text { et } 2006 \text { ) (2) }\end{array}$} \\
\hline & & \multicolumn{2}{|c|}{$\begin{array}{l}\text { Famille professionnelle } \\
\text { initiale }\end{array}$} & & \multicolumn{2}{|c|}{$\begin{array}{c}\text { Famille professionnelle } \\
\text { initiale }\end{array}$} \\
\hline & $\begin{array}{c}\text { (a) } \\
\text { Ensemble }\end{array}$ & $\begin{array}{c}\text { (b) } \\
\text { Agents } \\
\text { administratifs }\end{array}$ & $\begin{array}{c}\text { (c) } \\
\text { Techniciens }\end{array}$ & $\begin{array}{c}\text { (a) } \\
\text { Ensemble }\end{array}$ & $\begin{array}{c}\text { (b) } \\
\text { Agents } \\
\text { administratifs }\end{array}$ & $\begin{array}{c}\text { (c) } \\
\text { Techniciens }\end{array}$ \\
\hline $\begin{array}{l}\text { Genre } \times \text { temps plein (réf : } \\
\text { Homme temps plein) : }\end{array}$ & & & & & & \\
\hline $\begin{array}{l}\text { Femme temps plein } \\
\text { Femme temps partiel long } \\
\text { (> } 6 \text { mois) }\end{array}$ & $\begin{array}{c}\text { ns } \\
0,70\end{array}$ & $\begin{array}{c}\text { ns } \\
0,65\end{array}$ & $\begin{array}{l}\text { ns } \\
\text { ns }\end{array}$ & $\begin{array}{c}\text { ns } \\
0,41\end{array}$ & $\begin{array}{l}0,50 \\
0,25\end{array}$ & $\begin{array}{l}1,40 \\
\text { ns }\end{array}$ \\
\hline $\begin{array}{l}\text { Classe d'âge en } 1996 \\
\text { (réf : } 30-34 \text { ans) : } \\
<30 \text { ans } \\
35-39 \text { ans } \\
40-44 \text { ans } \\
45-49 \text { ans } \\
50-54 \text { ans }\end{array}$ & $\begin{array}{c}n s \\
0,76 \\
0,64 \\
0,50 \\
0,41\end{array}$ & $\begin{array}{c}n s \\
0,57 \\
0,42 \\
0,34 \\
0,23\end{array}$ & $\begin{array}{c}\text { ns } \\
0,74 \\
0,61 \\
0,44 \\
0,43\end{array}$ & $\begin{array}{l}1,77 \\
0,48 \\
0,37 \\
0,17 \\
0,10\end{array}$ & $\begin{array}{c}\text { ns } \\
0,54 \\
0,40 \\
0,20 \\
0,21\end{array}$ & $\begin{array}{l}1,76 \\
0,46 \\
0,34 \\
0,17 \\
0,10\end{array}$ \\
\hline $\begin{array}{l}\text { Diplôme : } \\
\text { BEPC/CEP } \\
\text { CAP/BEP } \\
\text { Bac }\end{array}$ & $\begin{array}{l}\text { ns } \\
\text { réf. } \\
\text { ns }\end{array}$ & $\begin{array}{l}\text { ns } \\
\text { réf. } \\
\text { ns }\end{array}$ & $\begin{array}{l}\text { ns } \\
\text { réf. } \\
\text { ns }\end{array}$ & $\begin{array}{l}1,52 \\
1,81 \\
1,86\end{array}$ & $\begin{array}{l}\text { ns } \\
\text { ns } \\
\text { ns }\end{array}$ & $\begin{array}{l}1,90 \\
2,03 \\
1,81\end{array}$ \\
\hline $\begin{array}{l}\mathrm{Bac}+2 \text { et plus } \\
\mathrm{Bac}+2 \\
\mathrm{Bac}+3 \text { et plus }\end{array}$ & ns & ns & ns & $\begin{array}{l}\text { réf. } \\
2,86\end{array}$ & $\begin{array}{l}\text { réf. } \\
2,37\end{array}$ & $\begin{array}{l}\text { réf. } \\
3,07\end{array}$ \\
\hline A été recruté(e) comme ouvrier & 1,13 & ns & 1,17 & 1,22 & ns & 1,22 \\
\hline
\end{tabular}




\begin{tabular}{|c|c|c|c|c|c|c|}
\hline & \multicolumn{6}{|c|}{ Tableau 5 (suite) } \\
\hline & \multicolumn{3}{|c|}{$\begin{array}{c}\text { Changer de coefficient au sein } \\
\text { des ETAM } \\
\text { (entre } 1997 \text { et 2006) (1) }\end{array}$} & \multicolumn{3}{|c|}{$\begin{array}{c}\text { Devenir cadre à partir des coefficients } \\
305 \text { et } 335 \\
\text { (entre } 1997 \text { et 2006) (2) }\end{array}$} \\
\hline & \multirow[b]{2}{*}{$\begin{array}{c}\text { (a) } \\
\text { Ensemble }\end{array}$} & \multicolumn{2}{|c|}{$\begin{array}{l}\text { Famille professionnelle } \\
\text { initiale }\end{array}$} & \multirow[b]{2}{*}{$\begin{array}{c}\text { (a) } \\
\text { Ensemble }\end{array}$} & \multicolumn{2}{|c|}{$\begin{array}{l}\text { Famille professionnelle } \\
\text { initiale }\end{array}$} \\
\hline & & \begin{tabular}{|c|} 
(b) \\
Agents \\
administratifs \\
\end{tabular} & $\begin{array}{c}\text { (c) } \\
\text { Techniciens }\end{array}$ & & $\begin{array}{c}\text { (b) } \\
\text { Agents } \\
\text { administratifs } \\
\end{array}$ & $\begin{array}{c}\text { (c) } \\
\text { Techniciens }\end{array}$ \\
\hline \multicolumn{7}{|l|}{ Coefficient initial : } \\
\hline 240 & 1,28 & ns & 1,38 & & & \\
\hline 255 & 1,19 & ns & 1,28 & & & \\
\hline 270 & réf. & réf. & réf. & & & \\
\hline 285 & 0,73 & ns & 0,81 & & & \\
\hline $\begin{array}{l}305 \\
335\end{array}$ & & & & $\begin{array}{l}\text { réf. } \\
6,75\end{array}$ & $\begin{array}{l}\text { réf. } \\
6,44\end{array}$ & $\begin{array}{l}\text { réf. } \\
7,40\end{array}$ \\
\hline \multicolumn{7}{|l|}{$\begin{array}{l}\text { Famille professionnelle initiale } \\
\text { (réf : techniciens): }\end{array}$} \\
\hline Agents administratifs & ns & & & 1,26 & & \\
\hline Commerciaux & ns & & & 2,55 & & \\
\hline Agents de Maîtrise & 1,56 & & & 1,64 & & \\
\hline $\begin{array}{l}\text { Établissement situé } \\
\text { en Région parisienne }\end{array}$ & ns & ns & ns & 0,60 & ns & 0,57 \\
\hline \multicolumn{7}{|l|}{ Nombre d'enfants (réf: deux) : } \\
\hline aucun & 0,85 & ns & 0,81 & ns & ns & ns \\
\hline un seul & ns & ns & ns & ns & ns & ns \\
\hline trois et plus & ns & ns & ns & ns & ns & ns \\
\hline \multicolumn{7}{|l|}{$\begin{array}{l}\text { Sur la période précédent } \\
\text { le changement de coefficient : }\end{array}$} \\
\hline $\begin{array}{l}\text { Naissance d'un enfant } \\
\text { Mobilité régionale }\end{array}$ & $\begin{array}{c}0,51 \\
\text { ns }\end{array}$ & $\begin{array}{c}0,65 \\
\text { ns }\end{array}$ & $\begin{array}{c}0,67 \\
\text { ns }\end{array}$ & $\begin{array}{c}0,76 \\
\text { ns }\end{array}$ & $\begin{array}{c}0,36 \\
\text { ns }\end{array}$ & $\begin{array}{l}\text { ns } \\
\text { ns }\end{array}$ \\
\hline $\begin{array}{l}\text { Changement de famille } \\
\text { professionnelle }\end{array}$ & 0,68 & 0,47 & 0,74 & 0,67 & ns & 0,78 \\
\hline Nombre d'observations & 3618 & 947 & 1845 & 3823 & 498 & 2803 \\
\hline $\begin{array}{l}\text { Pourcentage des observations } \\
\text { censurées à droite }\end{array}$ & $18,7 \%$ & $28,7 \%$ & $16,5 \%$ & $68,5 \%$ & $73,7 \%$ & $68,0 \%$ \\
\hline
\end{tabular}

ns : non significatif au seuil de $5 \%$; les populations (1) et (2) n'ayant pas la même structure d'éducation, les modalités de la variable diplôme ne sont pas les mêmes (d'où les cases grisées); dans les modèles (b) et (c) la variable «famille professionnelle initiale » n'est plus active puisque l'on se restreint à une seule de ces familles (d'où les cases grisées).

(1) Lecture : Sur l'ensemble des ETAM (employés, techniciens, agents de maîtrise), le risque instantané de promotion des femmes ayant pris un temps partiel long sur la période est égal à 0,70 fois le risque instantané de promotion des hommes travaillant à plein temps, en supposant ces deux populations en tous points identiques par ailleurs. Plus simplement, toutes choses égales par ailleurs, la vitesse de promotion au sein des coefficients ETAM des femmes ayant pris un temps partiel long est plus faible que celle des hommes travaillant à plein temps.

Champ : les ETAM dont le coefficient initial est compris entre 240 et 285 (présents en 1996 et 2006).

(2) Lecture : Sur l'ensemble des ETAM susceptibles de devenir cadres, le risque instantané de promotion des femmes ayant pris un temps partiel long sur la période est égal à 0,41 fois le risque instantané de promotion des hommes travaillant à plein temps, en supposant ces deux populations en tous points identiques par ailleurs. Plus simplement, toutes choses égales par ailleurs, la vitesse de promotion vers le statut Cadre des femmes ayant pris un temps partiel long est plus faible que celle des hommes travaillant à plein temps.

Champ : les ETAM dont le coefficient initial est égal à 305 ou 335 (présents en 1996 et 2006).

Source : fichiers RH de GFAuto. 


\section{Un effet sexe variable} selon la famille professionnelle

Dans l'ensemble de la population, toutes choses égales par ailleurs, on montre que le sexe ne suffit pas, en tant que tel, à expliquer les retards de promotion au sein des ETAM, ni même le passage cadre. De même, le nombre d'enfants n'aurait pas d'effet non plus : ni sur la vitesse de promotion des ETAM - ne pas en avoir constituerait même un handicap - ni sur la vitesse de passage cadre. Être une femme ayant pris un temps partiel long se révèlerait, en revanche, un facteur déterminant du retard de progression tant au sein des coefficients ETAM qu'aux portes du passage cadre. Une maternité ou une paternité récente, c'est-à-dire sur la période précédent le changement de coefficient ou le passage cadre, retarderait également la promotion. On en déduit un modèle de disponibilité, légitime pour l'entreprise, qui ne s'applique pas qu'aux seules femmes, mais dont elles pâtissent plus particulièrement puisque ce sont principalement elles qui aménagent leur temps de travail. On appelle « modèle de disponibilité » le fait d'être évalué sur sa présence sur le lieu de travail. De fait, l'amalgame entre engagement professionnel, performance et «disponibilité présentielle » est fortement répandu dans l'entreprise. Même s'il trouve de moins en moins d'écho auprès des plus jeunes, hommes ou femmes, qui aspirent à des modes de vie plus équilibrés, il constitue encore l'une des explications traditionnelles aux différences d'accès des deux sexes aux postes clés dans les entreprises (Laufer, 2001 ; Wajcman, 2003). Cette étude permet d'en mesurer les effets sur la vitesse de progression. Elle confirme également une explication plus subtile des retards de progression suggérée par Marry (2004) et mise en évidence par Pochic (2005), selon laquelle la réussite professionnelle dépendrait aussi de la configuration familiale. Ainsi, il ne suffit pas d'être disponible pour progresser rapidement ou accéder rapidement au statut de cadre, il faut aussi être « stable affectivement ». On trouve ici une explication plausible au retard de progression, relativement paradoxal, des ETAM sans enfants.

Les estimations sur les deux sous-populations, « agents administratifs » et « techniciens », révèlent que le sort des femmes en matière de promotion dépendrait également de leur famille profession- nelle d'origine. Contrairement à leurs consœurs des métiers administratifs, celles qui optent pour la filière technique semblent bénéficier non seulement d'un passage cadre plus rapide que leurs homologues masculins, mais également d'une relative neutralité des aménagements horaires auxquels elles peuvent avoir recours. Prime à la non-mixité ou simple effet de famille professionnelle? L'effet est complexe. $\mathrm{Au}$ sein des coefficients ETAM, on constate que la filière «maîtrise » est la plus rapide et, à l'inverse, que la filière administrative est la plus lente. On pourrait penser que le seul problème des femmes provient du fait qu'elles investissent majoritairement les métiers administratifs, moins valorisés dans un milieu industriel. Une réflexion complémentaire sur l'évaluation et la classification des emplois au sein de l'entreprise pourrait alors être préconisée (Lemière et Silvera, 2010). Mais cette explication ne tient que si les emplois administratifs sont systématiquement sous-valorisés. Or, concernant le passage cadre, toutes les familles professionnelles semblent permettre une promotion plus rapide que la filière technique. Les métiers techniques, majoritairement masculins, ne bénéficient donc pas systématiquement d'une «survalorisation »... ce sont plutôt les quelques femmes ayant opté pour la filière technique qui semblent survalorisées du fait de leur rareté.

L'effet d'un changement de famille professionnelle sur la vitesse de promotion confirme l'effet complexe de la famille professionnelle. Compte tenu de la prise de risque que cela représente, nous nous attendions à trouver un effet positif du changement de métier sur le rythme de progression. Or, le choix d'un changement de métier au sein de l'entreprise peut sans doute être rentable sur une période longue ; cependant, sur une période courte, nos résultats révèlent que cela tend à ralentir la vitesse de promotion. Sans doute une période d'apprentissage est-elle nécessaire à la maîtrise d'un nouveau métier, ce qui freinerait momentanément le rythme des promotions.

De même, nous nous attendions à retrouver, comme pour les cadres étudiés par ailleurs (Charbeau et Larquier 2007), un effet positif de la mobilité géographique. Cette pratique est, notons-le, beaucoup plus rare chez les ETAM que chez les cadres, mais elle n'a pas d'effet significatif sur la vitesse de promotion des ETAM, ni même sur le passage cadre. 
Pour progresser dans la hiérarchie des coefficients, le niveau de diplôme ne semble pas non plus constituer un atout majeur. En revanche, il devient un critère déterminant dans le passage cadre des techniciens. Toutes choses égales par ailleurs, plus le niveau de diplôme est élevé, plus la vitesse de promotion augmente. En revanche, la promotion interne ne semble pas freiner la promotion des techniciens : pour les deux types de promotion, les anciens ouvriers ont souvent progressé plus vite que les embauchés ETAM. Ceux qui ont été promus le seront encore. Pour les administratifs, plus rarement issus de la promotion interne, ce résultat n'est pas significatif.

Enfin, on retrouve un effet assez attendu de l'âge sur la vitesse de promotion : plus on est jeune, plus vite on progresse, soit en termes de coefficient, soit en termes de passage au statut cadre.

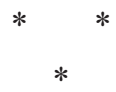

La motivation de la signature de l'accord d'égalité professionnelle, en novembre 2003, avait sa rationalité économique : étant donné sa nouvelle politique industrielle, la pénurie annoncée de main-d'œuvre qualifiée rendait GFAuto réceptif à la thématique de l'emploi féminin. Trois années plus tard, à l'aune des indicateurs prévus par la loi, l'évaluation de la politique se révèle très satisfaisante en termes d'équité entre femmes et hommes. Le cas de GFAuto est-il une success story alliant efficacité et équité ? Nous sommes plus prudentes. Alors que le RSC (rapport de situation comparée), outil de pilotage officiel de la politique menée, laisse apparaître une quasi-égalité de traitement entre les sexes, l'analyse détaillée des parcours suggère à l'inverse que les inégalités perdurent sous l'effet de trajectoires différenciées. En cause, ici, l'application, lors de l'évaluation des candidats à la promotion, d'un modèle de disponibilité, légitime d'un point de vue formel selon l'entreprise, mais en pratique défavorable aux femmes.

Une véritable égalité professionnelle devrait donc s'évaluer en termes de carrière et non pas seulement à coefficient égal, ce qui en quelque sorte est une condition nécessaire mais non suffisante. L'évaluation peut être plus ou moins sophistiquée, nécessitant de suivre une cohorte de salariés ou de la construire a posteriori. Mais elle ne peut faire l'économie d'une réflexion sur la mise en forme des données : doit-on raisonner à génération donnée ou à coefficient égal (et si oui, initial ou final)? Dans une perspective analytique ou de recherche, il nous semble que ce sont les promotions, ou plus généralement l'accès aux positions professionnelles, qu'on doit chercher à expliquer « toutes choses égales par ailleurs » et non les salaires. Sinon, le risque est de tomber dans le même travers : ignorer les inégalités qui se construisent avec le temps et qui sont masquées par les niveaux de qualification atteints qui figurent généralement dans une équation de salaire.

\section{Bibliographie}

Blau F., Devaro J. (2007), "New Evidence on Gender Differences in Promotion Rates: An empirical Analysis of a Sample of New Hires", Industrial Relations, Vol. 46, $\mathrm{n}^{\circ}$ 3, pp 511-550.

Booth A., Franscesconi M., Franck J. (2003), “A Sticky Floors Model of Promotion, Pay and Gender", European Economic Review, n 47, pp. 295-322.

Carrere M., Sabatier M., (2004), "Do female researchers face a glass ceiling in France? A hazard model of promotions", $14^{e}$ journée du SÉSAME, Séminaire d'Études et de Statistiques Appliquées à la Modélisation en Économie, Pau.

Carrere M., Sabatier M., Mangematin V. (2006), "Profiles of Academic Activities and Careers: Does Gender Matter? An Analysis based on French Life Scientist CV's", Journal of Technology Transfer, 31/3, pp 311-324.

Charbeau L. (2009), Inégalités de carrières entre hommes et femmes. Une approche par les conventions 
d'évaluation. Analyse des trajectoires des cadres et ETAM chez un constructeur automobile, Thèse pour le doctorat en sciences économiques, université Paris Ouest-Nanterre.

Charbeau L., Larquier G. de (2007), «Inégalités de salaires, inégalités d'évaluations : le cas des femmes cadres dans une grande entreprise industrielle», $27^{e s}$ journées de l'Association d'Économie Sociale, Nanterre, Septembre.

Coob-Clark D. (2001), "Getting Ahead: The Determinants of and Payoffs to Internal Promotions for Young U.S. Men and Women", in S.W. Polachek (eds), Worker Well-Being in a changing Labor Market, Research in Labor Economics, New York: JAI Elsevier Science, pp 339-372.

Cousin O. (2007), «La construction des inégalités hommes-femmes dans l'entreprise. Une analyse de cas dans la métallurgie », Sociologie du Travail, $\mathrm{n}^{\circ}$ 49, pp. 195-219.

Dupray A., Moullet S. (2004), «Disparités de carrières entre hommes et femmes au sein d'un marché interne : mobilités et salaires ", Relief Céreq, $\mathrm{n}^{\circ} 4$, pp. 55-72.

Fortino S. (2002), La mixité au travail, La Dispute, Paris.

Gadea C., Rezrari A. (2004), « Promotion et genre : une mosaïque de différences ", in Karvar A., Rouban L. (eds), Les cadres au travail, les nouvelles règles $d u$ jeu, pp 217-230, Paris, La Découverte.

Gerhart B.A., Milkovitch G.T. (1989), "Salaries, Salary Growth, and Promotions of Men and Women in a Large Private Firm", in Michael R.T., Hartmann H.I., O'Farrell B., Pay Equity: Empirical Inquiries, Washington DC: National Academy Press, pp 23-43.

Grésy B (2009), Rapport préparatoire à la concertation avec les partenaires sociaux sur l'égalité professionnelle entre les hommes et les femmes, rapport pour le ministre du Travail, des Relations sociales, de la Famille, de la Solidarité et de la Ville.
Laufer J. (2001), « Travail, carrières et organisation », in Laufer J., Marry M., Maruani M. (Dir.), Masculinféminin: questions pour les sciences de l'homme, pp. 57-80, Paris, PUF.

Lelièvre E., Bringé A. (1998), Manuel pratique pour l'analyse statistique des biographies, Collection «Méthodes et Savoirs », $n^{\circ}$ 2, INED-PUF.

Lemière S., Silvera R. (2010), Comparer les emplois entre les femmes et les hommes. De nouvelles pistes vers l'égalité professionnelle, Collection «Études et Recherches », HALDE.

Marry C. (2004), Une révolution respectueuse: les femmes ingénieures, Belin, Paris.

Mc Cue K. (1996), "Promotions and Wage Growth", Journal of Labor Economics, Vol. 14, $\mathrm{n}^{\circ} 2$, pp. 175-209.

Obka M. (2004), « L'accès des femmes aux métiers : la longue marche vers l'égalité professionnelle », Premières Synthèses, DARES, n 31.2 , juillet.

Pargamit M., Veum J. (1999), "What is a promotion?", Industrial and Labor relations Review, Vol. 52, $\mathrm{n}^{\circ} 4$, pp. 581-601.

Pekkarinen T., Vartiainen J. (2004), "Gender Differences in Job Assignment and Promotion on a complexity Ladder of Jobs", IZA Discussion Paper, $n^{\circ} 1184$.

Pochic S. (2005), «Faire carrière: l'apport d'une approche en termes de genre », Formation Emploi, $\mathrm{n}^{\circ} 91$, pp. 75-93.

Ranson G., Reeves W.J. (1996), “Gender, Earnings and Proportions of Women: Lessons from a HighTech Occupation", Gender and Society, Vol. 10, n 2, pp. 168-184.

Wajcman J. (2003), « Le genre au travail », in Laufer J., Marry M., Maruani M. (Ed.), Le travail du genre, Paris, La Découverte, pp. 151-162. 
Résumé

\title{
Évaluer une politique d'égalité professionnelle en entreprise : I'apport d'une analyse en termes de parcours
}

\author{
Lidwine Charbeau et Guillemette de Larquier
}

L'article porte sur l'évaluation d'une politique d'égalité professionnelle entre hommes et femmes au sein d'un groupe industriel du secteur automobile. Appréhender la dimension forcément longitudinale des carrières offertes aux hommes et aux femmes dans un marché interne s'avère alors un enjeu essentiel. Le Rapport de situation comparée, outil de pilotage voulu par le législateur, laisse apparaître une quasiégalité de traitement entre les sexes. Cependant, l'analyse détaillée des parcours suggère, à l'inverse, que les inégalités perdurent sous l'effet de trajectoires différenciées, en particulier dans les métiers très féminisés. À l'aide de modèles de durée, nous cherchons alors à cerner les déterminants des promotions au sein des coefficients ETAM (employés, techniciens, agents de maîtrise) et vers le statut de cadre.

Mots clés :

Division sexuelle du travail, inégalité salariale, politique d'entreprise, industrie automobile, cheminement professionnel, promotion

Journal of Economic Literature: J 16, M 51, J 31 